\title{
Diente supernumerario mesiodens en posición transversal invertida. Reporte de caso
}

\section{Mesiodens supernumerary tooth in inverted transverse position. A case report}

\author{
José Oliva Chumán* \\ Facultad de Odontología, \\ Universidad de San Martín de Porres, Perú
}

\section{Resumen}

Los dientes supernumerarios se presentan como dientes o estructuras similares, adicionales a los 20 dientes primarios y los 32 permanentes. $\mathrm{Su}$ etiología en su mayoría es considerada idiopática aunque también está asociada a dos síndromes que son la displasia cleidocraneal y la poliposis adenomatosa familiar. Parece haber evidencia que sugiere una predisposición familiar a producir dientes adicionales. Su presencia puede producir complicaciones como dientes impactados, erupción ectópica, desplazamiento de dientes permanentes ocasionando distopia o malposición dental, diastemas, que a su vez pueden producir problemas oclusales y funcionales. Pueden también presentarse quistes derivados de folículos supernumerarios. El tratamiento quirúrgico de exodoncia de los dientes supernumerarios es el tratamiento de elección siempre que sea posible, sin embargo, limitaciones como la edad del paciente, impactación o su ubicación pueden posponer la decisión de realizar el abordaje quirúrgico, por lo cual puede ser necesario realizar una fase de observación durante la etapa de dentición mixta, esperando una erupción de los dientes permanentes con las menores complicaciones.

Palabras clave: diente supernumerario; diente impactado; mesiodens

Este es un artículo Open Access bajo la licencia Creative Commons Atribución-NoComercial-Compartirlgual 4.0

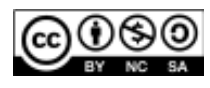




\begin{abstract}
Supernumerary teeth appear as teeth or structures similar and additional to the normal series of 20 primary and 32 permanent teeth. Their etiology is mostly idiopathic although it is also associated with two syndromes: cleidocranial dysplasia and familial adenomatous polyposis. There seems to be evidence suggesting a family tendency to produce extra teeth. Supernumerary teeth can result in complications such as impacted teeth, ectopic eruption, displaced permanent teeth causing dystopia or dental malposition, and diastemas, which in turn may generate occlusal and functional problems. Cysts derived from supernumerary follicles may also occur. Surgical extraction, i.e. exodontics, of supernumerary teeth is the treatment of choice whenever possible; however, limitations such as the patient's age, tooth impaction or tooth location may postpone the decision to perform the surgical approach. Thus, it may be necessary an observation phase during the mixed dentition stage to wait for the eruption of permanent teeth with the least complications
\end{abstract}

Keywords: supernumerary tooth; impacted tooth; mesiodens

\title{
Introducción
}

Los dientes supernumerarios representan una anomalía en el patrón de desarrollo y morfogénesis que ocurre en las zonas de soporte dentario de los maxilares ${ }^{(1)}$. Los dientes supernumerarios son dientes, o estructuras similares, adicionales a los 20 dientes primarios y los 32 permanentes ${ }^{(1)}$. Pueden ser únicos, múltiples, unilaterales o bilaterales en su distribución, erupcionados o impactados ${ }^{(2)}$. Pueden aparecer en cualquier zona de la dentición, aunque con frecuencia ocurren en la premaxila ${ }^{(1-3)}$. La región más común en la que surgen es entre los dos incisivos, por lo que toman el nombre de mesiodens, y cuya forma más común $(60 \%)$ es de tipo canino ${ }^{(4)}$.

Pippi ${ }^{(5)}$ evaluó la incidencia de supernumerarios en diferentes estudios, encontrando que es de 1.5\% (770 casos / 51130 pacientes examinados). Sin embargo, refiere que la incidencia informada fue bastante diferente de un 
estudio a otro, probablemente debido a diferencias en las muestras evaluadas (muestras escolares, muestras de odontología general, muestras de ortodoncia o pediátricas muestras de odontología) y en la evaluación. Los supernumerarios fueron más comunes en hombres que en mujeres $(67.5 \%$ versus $32.5 \%)$ con una proporción de 1 entre $0.48(1026 / 495){ }^{(5)}$. En una muestra seleccionada de 1176 casos, se produjo un supernumerario en el $72.6 \%$, dos supernumerarios en el $23 \%$ y 3 supernumerarios o más en el $4.4 \%$ (5). El 85\% de los supernumerarios se ubicaron en el maxilar superior, mientras que el 15\% estaba en la mandíbula. El área incisiva-canina superior estuvo involucrada en la gran mayoría de casos $(69.5 \%$; 1157 casos); en la mandíbula, afectaron con mayor frecuencia el área premolar (42\%) que representaba el segundo más localización frecuente en la boca. La ubicación palatina/lingual mostró una mayor incidencia (77.2\%) que las ubicaciones bucales y de la línea media $(7.8 \%$ y $15 \%)$. La mayor incidencia del tipo cónico es evidente $(56.6 \%)$ respecto a otras formas ${ }^{(5)}$. La mayoría de los dientes supernumerarios son idiopáticos, pero también se producen en síndromes genéticos, donde pueden ser una clave diagnóstica importante ${ }^{(6)}$. Los dos síndromes con los que se asocia comúnmente son la displasia cleidocraneal y la poliposis adenomatosa familiar. Los trastornos del desarrollo menos frecuentes son el síndrome de Gardner, el síndrome de Fabry Anderson, la displasia condroectodérmica (síndrome de Ellis-van Greveld), la incontinencia pigmentaria y el síndrome trico-rino-falangeal (7). En casos en los que el diagnóstico no es oportuno o en los que no se puede realizar el tratamiento quirúrgico de exodoncia, se pueden presentar las siguientes complicaciones: Su presencia puede producir complicaciones como dientes impactados, erupción ectópica, desplazamiento de dientes permanentes ocasionando distopia o malposición dental, diastemas, que a su vez pueden producir problemas oclusales y funcionales, interferencias con tratamientos de ortodoncia, diastemas. Pueden presentarse también quistes derivados de folículos supernumerarios, caries de dientes vecinos debido al aumento de retención de placa dental causada por el diente supernumerario, rizólisis, pérdida de vitalidad dental, diagnóstico diferencial con odontoma, tumor adenomatoide, cementoblastoma ${ }^{(8-10)}$. 


\section{Reporte de caso}

El paciente J. R. O., de sexo masculino, de 6 años de edad, acudió junto a sus padres a la clínica odontológica especializada. Durante el examen clínico se observó una pieza dentaria que se encontraba erupcionando con notable discromía y alteración de la morfología a nivel del incisivo central superior derecho permanente (Figura 1), se procedió a la toma de una radiografía panorámica (Figura 2), determinando el diagnóstico de diente supernumerario (mesiodens), por lo cual se procedió a la exodoncia del supernumerario pues impedía la erupción incisivo central superior derecho permanente (Figura 3). Sin embargo, en la tomografía cone beam, se aprecia también otro diente supernumerario mesiodens en posición transversal invertida, es decir, la porción apical en sentido vestibular y la porción coronal en sentido palatino (Figuras 4, 5 y 6). Se optó por realizar una observación del caso durante el periodo de erupción de los incisivos centrales superiores con la finalidad de monitorizar que la erupción se produzca sin complicaciones, dado que ese diente se encuentra impactado muy cerca al piso de las fosas nasales y por la edad del paciente que es muy pequeño. Si se presentara alguna de las complicaciones por dientes supernumerarios, mencionadas previamente en este artículo, se evaluaría realizar un procedimiento quirúrgico de exodoncia del diente supernumerario y procedimientos correctivos que dependen del alcance de las complicaciones presentadas, como por ejemplo, el tratamiento de ortodoncia.

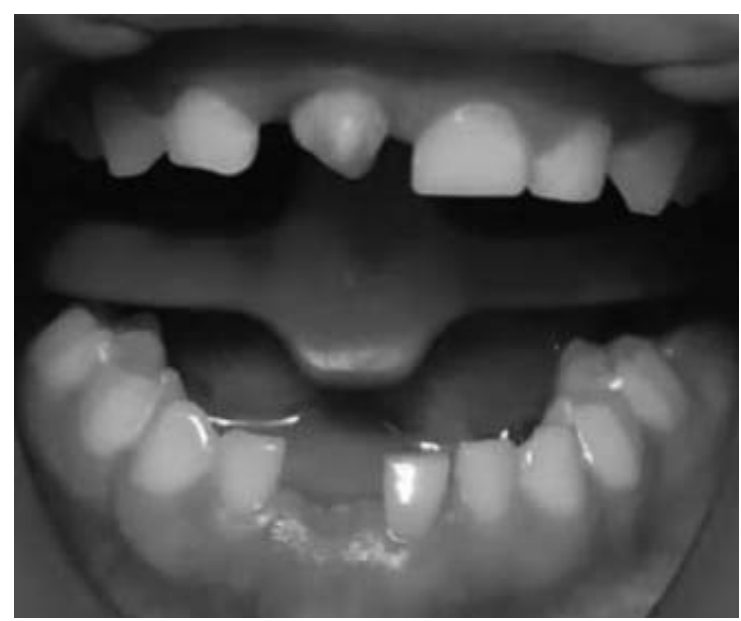

Figura 1. Diente supernumerario a nivel de 11. 


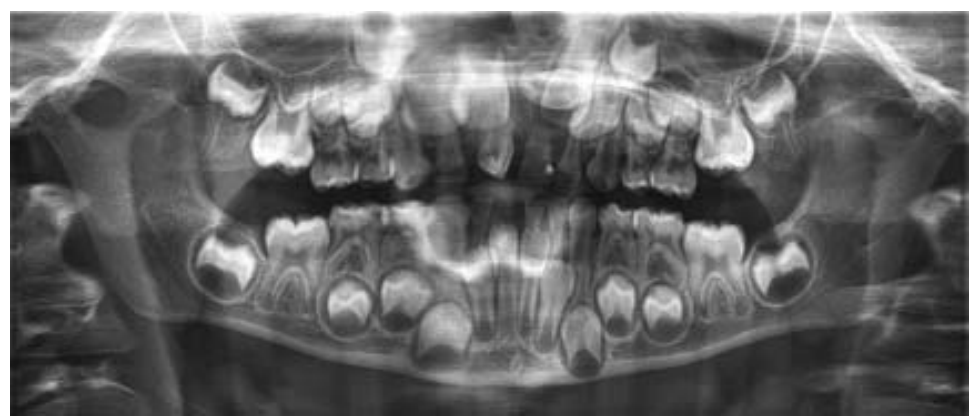

Figura 2. Diente supernumerario a nivel de 11.

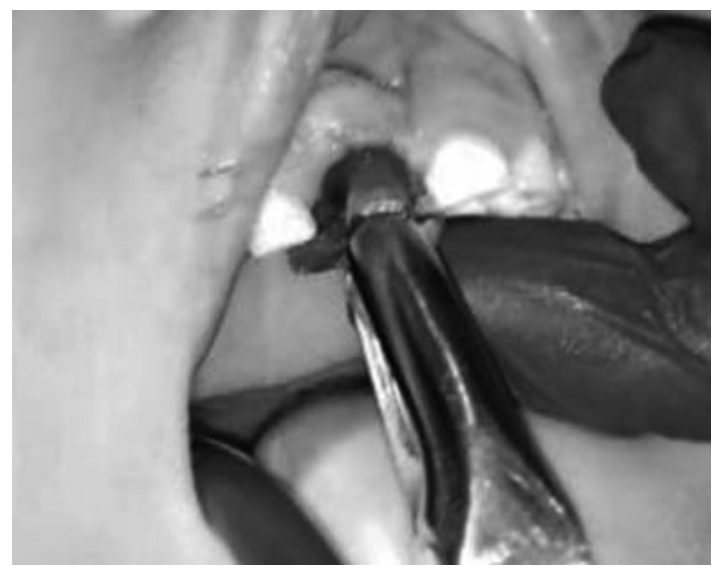

Figura 3. Exodoncia de diente supernumerario.

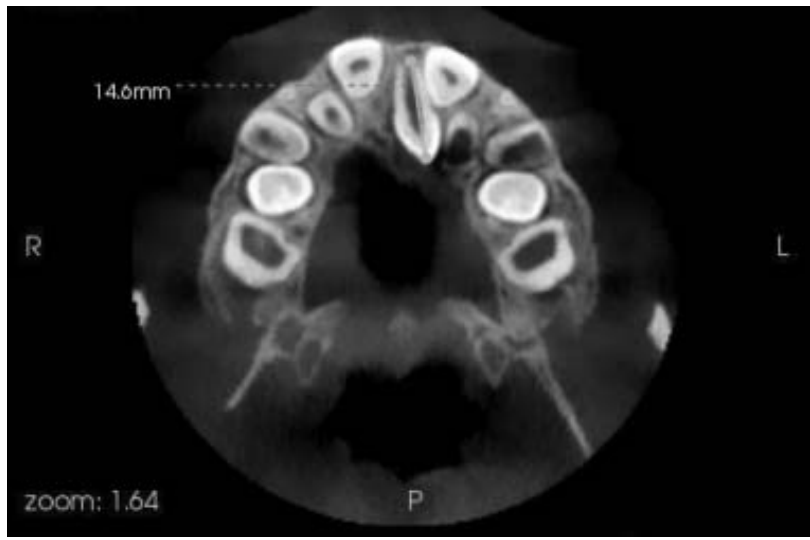

Figura 4. Corte tomográfico axial que muestra incisvos centrales permanentes y mesiodens impactado transversal invertido. 


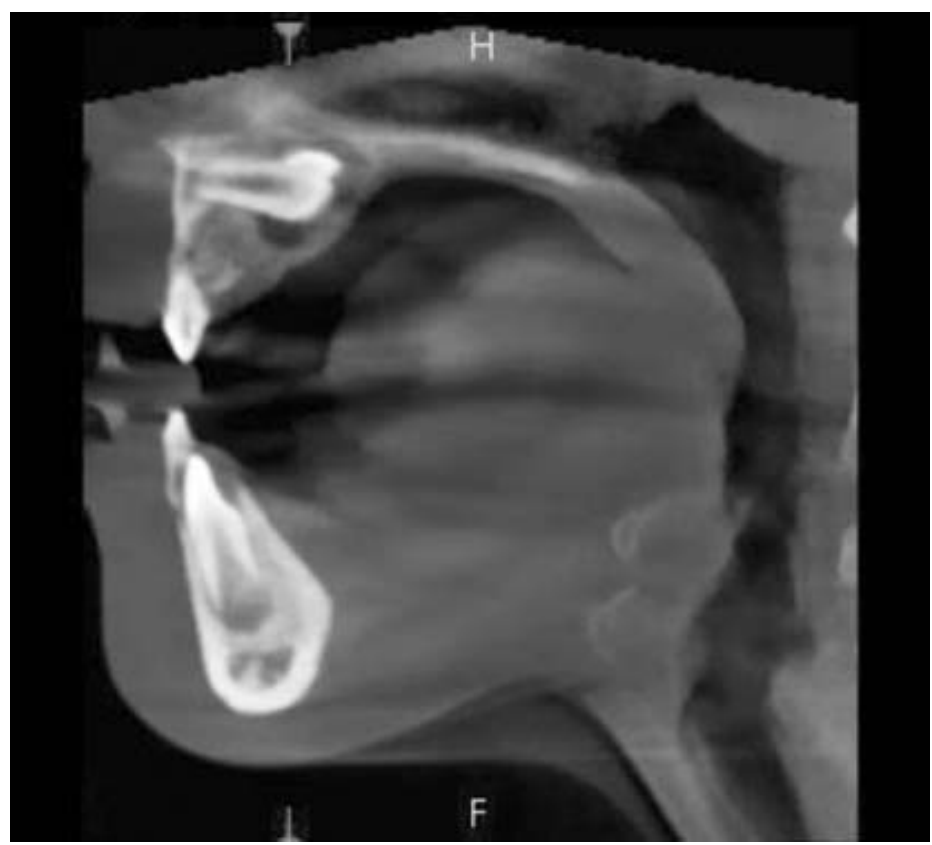

Figura 5. Corte tomográfico sagital que muestra mesiodens impactado transversal invertido.

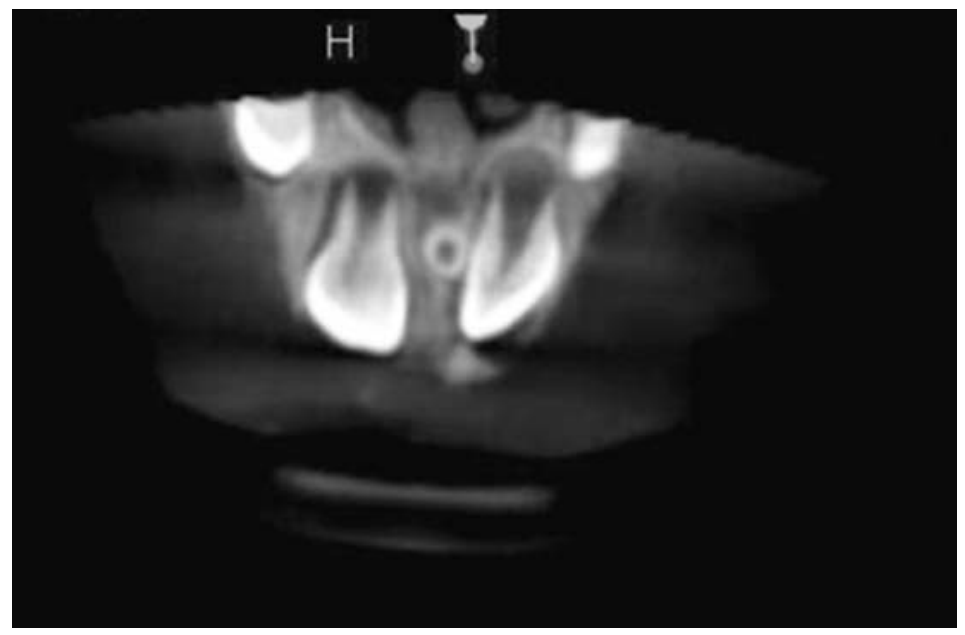

Figura 6. Corte tomográfico coronal que muestra incisvos centrales permanentes y mesiodens impactado transversal invertido a nivel vestibular. 


\section{Discusión}

Los dientes supernumerarios pueden aparecer en dentición primaria y permanente de niños y adolescentes. Respecto a su etiología, también ha sido atribuido a patrones hereditarios (genéticos). Se han propuesto varias teorías para la etiología de la hiperdoncia o dientes supernumerarios: teoría de la diferenciación, teoría de la concrescencia, teoría postpermanente, teoría de la dicotomía y teoría de la hiperactividad. Sin embargo, la teoría de la hiperactividad, que establece que los dientes supernumerarios se derivan de la hiperactividad local independiente de la lámina dental, ha sido la más aceptada. De acuerdo con esta hipótesis, la extensión lingual de un diente dental adicional conduce a un mesiodens eumórfico, mientras que la forma rudimentaria surge de la proliferación de restos epiteliales de la lámina dental inducida por la presión de la dentición ${ }^{(11,12)}$. Parece haber evidencia que sugiere una predisposición familiar a producir dientes adicionales. Muchos casos publicados de dientes supernumerarios mencionan la reincidencia dentro de la misma familia, pero sin investigar este aspecto en detalle ${ }^{(13)}$. Muchos autores investigaron el componente genético de la presencia de dientes supernumerarios, mencionando que es un rasgo autosómico dominante con falta de penetrancia en algunas generaciones, incluso que podría ser una herencia ligada al sexo por su predominancia en hombres sobre mujeres, refutando la teoría de la mutación, sugiriendo que la herencia familiar es un factor clave en la presencia de mesiodens ${ }^{(14-16)}$.

Estos dientes son importantes debido a su asociación con la posición, erupción y alteraciones de retención de dientes permanentes. Su hallazgo depende de un diagnóstico completo y oportuno para determinar su presencia, la formación de sus raíces y ubicación. En el presente caso, el tratamiento fue realizado en un niño de 06 años de edad, se decidió la exodoncia del diente supernumerario erupcionado debido a que impedía la erupción del diente permanente, mientras que se decidió observar mediante controles periódicos la exfoliación del incisivo central superior izquierdo y la erupción de los incisivos centrales superiores permanentes, esperando que el supernumerario impactado no produzca problemas de impactación, desviación, giroversión o apiñamiento en ellos. 


\section{Referencias}

1. Anthonappa RP, King NM, Rabie, ABM. Aetiology of supernumerary teeth: a literature review. Eur Arch Paediatr Dent. 2013;14(5):279-88; PMID: 24068489; https://doi.org/10.1007/s40368-013-0082-z

2. Garvey MT, Barry HJ, Blake M. Supernumerary teeth-an overview of classification, diagnosis and management. J Can Dent Assoc. 1999;65:612-6; PMID: 10658390

3. Rajab LD, Hamdan MAM. Supernumerary teeth: review of the literature and a survey of 152 cases. Int $\mathrm{J}$ Paediatr Dent. 2002;12:244-54; PMID: 12121534; https://doi.org/10.1046/j.1365-263X.2002.00366.X

4. Colak H, Uzgur R, Tan E, Hamidi MM, Turkal M, Colak T. Investigation of prevalence and characteristics of mesiodens in a non-syndromic 11256 dental outpatients. Eur Rev Med Pharmacol Sci. 2013;17:2684-9; PMID: 24142619

5. Pippi R. Odontomas and supernumerary teeth: is there a common origin? Int J Med Sci. 2014;11:1282-97.

6. Wijn MA, Keller JJ, Giardiello FM, Brand HS. Oral and maxillofacialmanifestations of familial adenomatous polyposis. Oral Dis. 2007;13(4):360-5.

7. Lubinsky M, Kantaputra PN.Syndromes with supernumerary teeth. Am J Med Genet Part. 2016;170(A):261116.

8. Gallas MM, García A. Case study: retention of permanent incisors by mesiodens: a family affair. Br Dent J. 2000;188(2):63-4.

9. Contreras Somoza MF, Salinas Noyola A, Sáez Martínez S, Sellet LG. Signos de dientes supernumerarios. Rev Op Dent Endod. 2007;5(60):210.

10. Oropeza MP. Supernumerary teeth. Clinical case report. Revista Odontológica Mexicana. 2013;17(2):90-4

11. Sykaras SN. Mesiodens in primary and permanent dentition. Oral Surg. 1975;39:870-874.

12. Pindborg JJ. Pathology of Dental Hard Tissues. Philadelphia: W. B. Saunders Company. 1970;26-33.

13. Rubin MM, Nevins A, Berg M, Borden B. A comparison of identical twins in relation to three dental anomalies: multiple supernumerary teeth, juvenile periodontosis, and zero caries incidence. Oral Sur 1981;52:39194.

14. Sedano HO, Gorlin RJ. Familial occurrence of mesiodens. Oral Surg Oral Med Oral Pathol. 1969;27(3):3602.

15. Stellzig A, Basdra EK, Komposch G. Mesiodentes: incidence, morphology, etiology. J Orofac Orthop 1997;58(3):144-53.

16. Cadenat $\mathrm{H}$, Combelles R, Fabert G, Clouet M. Mesiodens and heredity (Mesiodens et heredite). Rev Stomatol Chir Maxillofac 1977;78(5):341-6. 